\title{
Respiratory Variations in Pulse Pressure Reflect Central Hypovolemia during Noninvasive Positive Pressure Ventilation
}

\author{
Ingrid Elise Hoff, ${ }^{1,2}$ Lars Øivind Høiseth, ${ }^{2,3}$ Jonny Hisdal, ${ }^{4}$ Jo Røislien, ${ }^{1,5}$ \\ Svein Aslak Landsverk, ${ }^{2}$ and Knut Arvid Kirkebøen ${ }^{2,3}$ \\ ${ }^{1}$ Norwegian Air Ambulance Foundation, Holterveien 24, 1441 Drøbak, Norway \\ ${ }^{2}$ Department of Anaesthesiology, Oslo University Hospital, P.O. Box 4956, Nydalen, 0424 Oslo, Norway \\ ${ }^{3}$ Faculty of Medicine, University of Oslo, P.O. Box 1072 Blindern, 0316 Oslo, Norway \\ ${ }^{4}$ Department of Vascular Medicine, Oslo University Hospital, P.O. Box 4956, Nydalen, 0424 Oslo, Norway \\ ${ }^{5}$ Department of Biostatistics, Institute of Basic Medical Sciences, University of Oslo, P.O. Box 1072 Blindern, 0316 Oslo, Norway
}

Correspondence should be addressed to Ingrid Elise Hoff; iehoff@yahoo.no

Received 14 September 2013; Revised 9 December 2013; Accepted 22 December 2013; Published 19 February 2014

Academic Editor: Djillali Annane

Copyright (C) 2014 Ingrid Elise Hoff et al. This is an open access article distributed under the Creative Commons Attribution License, which permits unrestricted use, distribution, and reproduction in any medium, provided the original work is properly cited.

\begin{abstract}
Background. Correct volume management is essential in patients with respiratory failure. We investigated the ability of respiratory variations in noninvasive pulse pressure $(\triangle \mathrm{PP})$, photoplethysmographic waveform amplitude $(\Delta \mathrm{POP})$, and pleth variability index (PVI) to reflect hypovolemia during noninvasive positive pressure ventilation by inducing hypovolemia with progressive lower body negative pressure (LBNP). Methods. Fourteen volunteers underwent LBNP of $0,-20,-40,-60$, and $-80 \mathrm{mmHg}$ for $4.5 \mathrm{~min}$ at each level or until presyncope. The procedure was repeated with noninvasive positive pressure ventilation. We measured stroke volume (suprasternal Doppler), $\triangle \mathrm{PP}$ (Finapres), $\triangle \mathrm{POP}$, and PVI and assessed their association with LBNP-level using linear mixed model regression analyses. Results. Stroke volume decreased with each pressure level $(-11.2 \mathrm{~mL}, 95 \% \mathrm{CI}-11.8,-9.6, P<0.001)$, with an additional effect of noninvasive positive pressure ventilation $(-3.0 \mathrm{~mL}, 95 \% \mathrm{CI}-8.5,-1.3, P=0.009)$. $\Delta \mathrm{PP}$ increased for each LBNP-level $(1.2 \%, 95 \%$ CI $0.5,1.8, P<0.001)$ and almost doubled during noninvasive positive pressure ventilation (additional increase $1.0 \%, 95 \% \mathrm{CI} 0.1,1.9, P=0.003)$. Neither $\triangle \mathrm{POP}$ nor PVI was significantly associated with LBNP-level. Conclusions. During noninvasive positive pressure ventilation, preload changes were reflected by $\triangle \mathrm{PP}$ but not by $\triangle \mathrm{POP}$ or PVI. This implies that $\triangle \mathrm{PP}$ may be used to assess volume status during noninvasive positive pressure ventilation.
\end{abstract}

\section{Introduction}

Pulse pressure variations $(\triangle \mathrm{PP})$ reflect volume status during mechanical ventilation [1]. Respiratory variations in the photoplethysmographic waveform amplitude $(\triangle \mathrm{POP})$ and the pleth variability index (PVI) are proposed as noninvasive alternatives [2,3]. In spontaneously breathing subjects, only passive leg raise and the end-expiratory occlusion test have been shown to consistently reflect preload dependency $[4,5]$. However, the literature is divided on two major issues concerning the usefulness of dynamic variables: whether they are applicable during spontaneous breathing $[6,7]$ and whether the photoplethysmographic waveform derived variables are useful alternatives to pulse pressure variation $[8,9]$. Due to this uncertainty, the applicability of dynamic variables is currently limited to patients on controlled mechanical ventilation. However, with increasing data on negative consequences of intubation and mechanical ventilation, noninvasive positive pressure ventilation (NPPV) is frequently used in emergency departments and intensive care units. Patients treated with NPPV are often on the verge of respiratory failure, and correct fluid management is essential.

The aim of the present study was therefore to explore the ability of dynamic variables to reflect graded hypovolemia during NPPV. Lower body negative pressure (LBNP) is a well-established model for central hypovolemia and preload reduction $[10,11]$. This noninvasive model in healthy volunteers enables investigation of arterial pressure and 
Spontaneous breathing

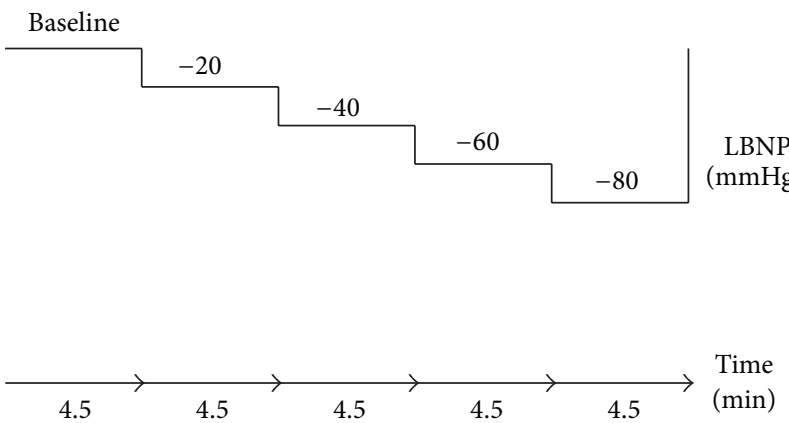

Noninvasive positive pressure ventilation
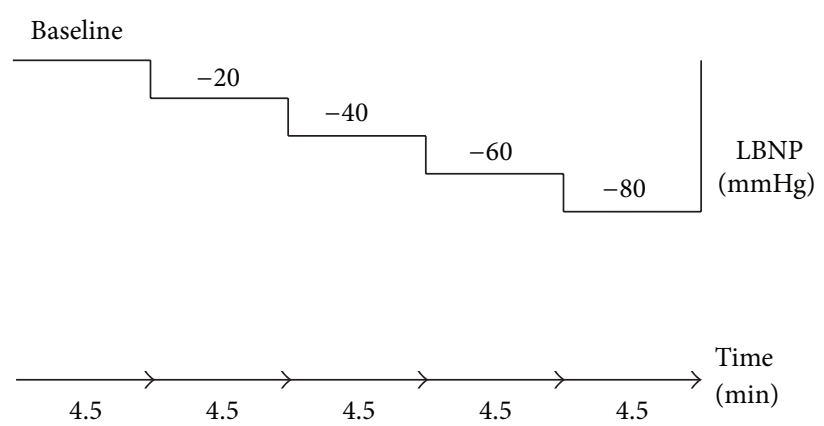

FIGURE 1: Schematic illustration of the experimental protocol. Each level was kept for $4.5 \mathrm{~min}$. LBNP: lower body negative pressure.

photoplethysmographic waveform derived variables without pain or medication, factors which could influence the results. We hypothesized that the reduction in stroke volume (SV) induced by increasing levels of LBNP would be aggravated with the application of NPPV and that the reduction in SV would be reflected in the dynamic variables $\triangle \mathrm{PP}, \triangle \mathrm{POP}$, and PVI.

\section{Materials and Methods}

After approval by the regional ethics committee (REK SørØst, ref.no 2009/2180, December 2009), written informed consent was obtained from 14 healthy volunteers ( 7 male, 7 female, aged $28 \pm 7$ years, height $177 \pm 10 \mathrm{~cm}$, and weight $71 \pm$ $13 \mathrm{~kg}($ mean $\pm \mathrm{SD}))$. The subjects were instructed to refrain from alcohol or caffeinated drinks 24 hours prior to participation. Pregnant women and subjects using cardiovascular medication were not included.

2.1. Experimental Protocol. Subjects were in the supine position during experiments, which were performed in room temperature. LBNP was applied by a custom made LBNPchamber previously described [12] and induced by stepwise suction of air out of the chamber. After baseline measurements, subjects underwent consecutive LBNP-pressures of $-20,-40,-60$, and $-80 \mathrm{mmHg}$. Each level was kept for $4.5 \mathrm{~min}$. After minimum $15 \mathrm{~min}$ rest, the procedure was repeated with NPPV. NPPV was applied via a face mask with intermittent positive pressure (IPPV-mode), tidal volume $10 \mathrm{~mL} / \mathrm{kg}$ ideal weight, positive end-expiratory pressure $(\mathrm{PEEP})=0 \mathrm{~cm} \mathrm{H} \mathrm{H}_{2} \mathrm{O}$, fraction of inspired oxygen 0.21 , and respiratory frequency of 10-12/min (Evita 4, Dräger Medizintechnik GmbH, Lübeck, Germany). Spontaneous breathing and mask leakage were minimized by thorough mask adjustment and by ensuring compliance with the ventilation mode before data recordings. The protocol was discontinued if one of the following events occurred: systolic blood pressure $<70 \mathrm{mmHg}$, a sudden decrease in systolic blood pressure $\geq 15 \mathrm{mmHg}$, a decrease in heart rate $(\mathrm{HR}) \geq 15$ beats $/ \mathrm{min}$, dizziness, sweating, or nausea. The experimental protocol is illustrated in Figure 1.
2.2. Data Acquisition and Analysis. Data were recorded over the total interval of each LBNP-level, that is, $4.5 \mathrm{~min}$. Data from all completed LBNP-levels are included in the analysis. Calculations were made from data sampled and averaged over 10 consecutive respiratory cycles without arrhythmia. Respiratory movements were recorded with a custom-made air flowmeter. During NPPV tidal volume, respiratory rate, airway pressures, leakage, and spontaneous breathing activity were continuously measured and recorded every $10 \mathrm{~s}$ using commercial software (VentView 2.0, Dräger Medical Ag \& Co, Lübeck, Germany). In order to investigate the effects of NPPV the data was manually filtered after recording and 10 consecutive respiratory cycles $(1 \mathrm{~min})$ without excessive spontaneous breathing ( $>5 \%$ of the corresponding minute volume per respiratory cycle) were identified. Hemodynamic measurements from the corresponding minute were then analyzed. $\triangle \mathrm{PP}$ and $\triangle \mathrm{POP}$ were calculated in a custom made program (Labview 8.2; National Instruments, TX, USA), according to Michard [13]. One respiratory cycle was manually delimitated and the program displayed corresponding blood pressure and photoplethysmographic waveform curves. The photoplethysmographic waveform, PVI, and PI were obtained from a finger clip (Masimo Radical 7, version 7.3.1.1, Masimo Corp., Irvine, CA, USA) on the third finger of the right hand, which was covered to prevent temperature loss and disturbance of the signal from ambient light. PVI and PI were calculated according to the manufacturer's algorithms (http://www.masimo.com/pdf/ whitepaper/LAB4583A.pdf; 13.09.2013). Averaging period for PI was set to $2 \mathrm{~s}$ in the pulse oximeter. PVI and PI were downloaded using the TrendCom software (Masimo) and averaged over $1 \mathrm{~min}$. Continuous arterial pressure was obtained noninvasively at heart level from the left third finger (Finometer, FMS Finapres Medical Systems BV, Amsterdam, The Netherlands). SV was obtained continuously with suprasternal Doppler (SD-100, GE Vingmed Ultrasound, Horten, Norway) by an experienced operator. An angle of $20^{\circ}$ and a diameter of the aortic valve of $20 \mathrm{~mm}$ were assumed in the calculation of SV from the velocity-time integrals. Heart rate (HR) was obtained from a standard 3-lead electrocardiogram (ECG). ECG, arterial pressure, and photoplethysmographic waveforms were sampled at $400 \mathrm{~Hz}$. 
TABLE 1: Dynamic variables and hemodynamic data during spontaneous breathing and noninvasive positive pressure ventilation.

\begin{tabular}{|c|c|c|c|c|c|c|c|c|c|c|}
\hline \multirow[t]{2}{*}{ LBNP-level } & \multicolumn{2}{|c|}{$\begin{array}{l}\text { Subjects completing } \\
\text { the LBNP-level }(n)\end{array}$} & \multicolumn{2}{|c|}{$\Delta \mathrm{PP}(\%)$} & \multicolumn{2}{|c|}{$\triangle \mathrm{POP}(\%)$} & \multicolumn{2}{|c|}{ PVI (\%) } & \multicolumn{2}{|c|}{ PI (\%) } \\
\hline & SB & NPPV & SB & NPPV & SB & NPPV & SB & NPPV & SB & NPPV \\
\hline Baseline & 14 & 12 & $7.4 \pm 3.1$ & $7.1 \pm 2.4$ & $12.4 \pm 5.4$ & $13.5 \pm 10.0$ & $18.5 \pm 6.9$ & $18.4 \pm 10.0$ & $2.8 \pm 2.1$ & $3.2 \pm 2.0$ \\
\hline 20 & 14 & 12 & $7.6 \pm 2.5$ & $7.1 \pm 1.9$ & $12.7 \pm 6.1$ & $14.0 \pm 7.5$ & $18.8 \pm 8.0$ & $17.8 \pm 11.6$ & $2.4 \pm 1.9$ & $2.5 \pm 1.4$ \\
\hline 40 & 13 & 10 & $8.3 \pm 8.0$ & $9.8 \pm 4.4$ & $13.4 \pm 8.0$ & $14.4 \pm 6.7$ & $18.6 \pm 7.2$ & $20.1 \pm 10.1$ & $2.2 \pm 1.6$ & $2.7 \pm 1.4$ \\
\hline 60 & 12 & 10 & $9.3 \pm 3.8$ & $12.1 \pm 5.2$ & $12.2 \pm 4.1$ & $14.4 \pm 6.2$ & $21.1 \pm 8.3$ & $22.1 \pm 9.9$ & $2.3 \pm 1.4$ & $2.5 \pm 1.1$ \\
\hline 80 & 11 & 9 & $12.6 \pm 7.1$ & $15.7 \pm 6.0$ & $16.6 \pm 5.5$ & $18.4 \pm 6.6$ & $22.5 \pm 8.5$ & $26.9 \pm 10.4$ & $2.1 \pm 1.2$ & $2.1 \pm 1.3$ \\
\hline \multirow[t]{2}{*}{ LBNP-level } & \multicolumn{2}{|c|}{$\begin{array}{l}\text { Subjects completing } \\
\text { the LBNP-level }(n)\end{array}$} & \multicolumn{2}{|c|}{$\mathrm{SV}(\mathrm{mL})$} & \multicolumn{2}{|c|}{ HR (beats/min) } & \multicolumn{2}{|c|}{ MAP $(\mathrm{mm} \mathrm{Hg})$} & \multicolumn{2}{|c|}{ PP (mm Hg) } \\
\hline & SB & NPPV & SB & NPPV & SB & NPPV & SB & NPPV & SB & NPPV \\
\hline Baseline & 14 & 12 & $80 \pm 13$ & $79 \pm 12$ & $59 \pm 8$ & $57 \pm 6$ & $78 \pm 11$ & $78 \pm 17$ & $58 \pm 12$ & $62 \pm 9$ \\
\hline 20 & 14 & 12 & $73 \pm 14$ & $67 \pm 14$ & $58 \pm 8$ & $59 \pm 8$ & $76 \pm 11$ & $75 \pm 18$ & $60 \pm 14$ & $60 \pm 17$ \\
\hline 40 & 13 & 10 & $60 \pm 13$ & $53 \pm 15$ & $64 \pm 9$ & $67 \pm 11$ & $77 \pm 13$ & $76 \pm 20$ & $57 \pm 14$ & $55 \pm 17$ \\
\hline 60 & 12 & 10 & $50 \pm 13$ & $43 \pm 11$ & $73 \pm 11$ & $78 \pm 14$ & $77 \pm 12$ & $77 \pm 19$ & $51 \pm 11$ & $52 \pm 16$ \\
\hline 80 & 11 & 9 & $35 \pm 12$ & $32 \pm 8$ & $89 \pm 17$ & $93 \pm 20$ & $77 \pm 14$ & $72 \pm 23$ & $45 \pm 9$ & $45 \pm 19$ \\
\hline
\end{tabular}

Data are mean \pm SD. LBNP: lower body negative pressure; SB: spontaneous breathing; NPPV: noninvasive positive pressure ventilation; $\triangle$ PP: pulse pressure variation; $\triangle$ POP: photoplethysmographic waveform variation; PVI: pleth variability index; PI: perfusion index; HR: heart rate; SV: stroke volume; MAP: mean arterial pressure; PP: pulse pressure.

2.3. Statistics. The primary endpoint was the change in $\Delta \mathrm{PP}$, $\triangle \mathrm{POP}, \mathrm{PVI}$, and PI following the transition from spontaneous breathing to noninvasive positive pressure ventilation. Data are given as mean \pm SD unless otherwise stated. The associations between the independent variables LBNP and NPPV and the dependent variables $\mathrm{HR}$, mean arterial pressure (MAP), SV, $\triangle \mathrm{PP}, \triangle \mathrm{POP}, \mathrm{PVI}$, and PI were analyzed by linear mixed model regression analyses. Linear mixed model is a generalization of traditional linear regression, which adjusts for the correlation between repeated measurements within each subject and finds the best linear fit to the data across all individuals. The model maximizes power by utilizing all data despite missing observations following the premature termination of the LBNP-protocol in some subjects. The effect estimates describe the mean effect of LBNP on the hemodynamic variables when going from one LBNP-level to the next. The difference in hemodynamic variables with and without NPPV was assessed by adding the interaction term $\mathrm{LBNP} * \mathrm{NPPV}$ in the regression model. Introduction of an interaction term is necessary where the effect of one variable (LBNP) is affected by the presence or value of another variable (NPPV). Results are given as coefficients (beta-values) with 95\% confidence intervals (CI). $P$ values below 0.05 were considered statistically significant. As no similar studies had previously been published, a conventional power analysis was not performed. Due to the experimental nature of the study, the number of study subjects was limited to 14 . Statistical calculations were performed in SPSS 19.0 (SPSS Inc.) Chicago, IL, USA) and R 2.12 (R Foundation for Statistical Computing, 2011).

\section{Results}

The number of subjects completing each level is shown in Table 1. Two subjects failed to comply with NPPV despite successful testing but completed the LBNP-series during spontaneous breathing. Data from these two subjects are included in the analysis of the effect of LBNP on hemodynamic variables. Hemodynamic data are summarized in Table 1 and mean values shown in Figure 2. Results from the linear mixed model analyses are given in Table 2. Different effects of LBNP and NPPV on hemodynamic variables are illustrated in Figure 3.

SV decreased significantly with progressive levels of LBNP, with a mean reduction of $11.2 \mathrm{~mL}$ (95\% CI -11.8, -9.6, $P<0.001)$ between each LBNP-level. After application of NPPV, SV was significantly lower at all LBNP-levels $(-3.0 \mathrm{~mL}$, 95\% CI $-8.5,-1.3, P=0.009)$ compared to spontaneous breathing (Figure 3(b)). $\triangle \mathrm{PP}$ was significantly affected both by LBNP-level alone and by the interaction between LBNP and NPPV (Figure $3(\mathrm{c})$ ). Whereas $\triangle \mathrm{PP}$ increased by $1.2 \%$ (95\% CI 0.5 1.8, $P<0.001$ ) between each LBNP-level during spontaneous breathing, the application of NPPV led to an additional increase of $1.0 \%$ ( $95 \%$ CI $0.1,1.9, P=0.033$ ) during LBNP, almost a doubling. NPPV alone did not affect $\triangle \mathrm{PP}$ significantly, meaning that NPPV only led to an increase in $\triangle \mathrm{PP}$ during hypovolemia induced by LBNP. Neither LBNP nor NPPV altered $\triangle \mathrm{POP}$ or PVI significantly. PI decreased significantly with progressive LBNP-levels, but was not affected by NPPV. Heart rate was significantly affected by LBNP alone. MAP did not change significantly during the LBNP-protocol or following the transition from spontaneous breathing to NPPV (Figure 3(a)). Pulse pressure decreased significantly between each LBNP-level $(-3.5 \mathrm{mmHg}, 95 \% \mathrm{CI}$ $-4.5,-2.0, P<0.001)$ but was unaffected by NPPV.

\section{Discussion}

The main finding in this experimental study is that $\Delta \mathrm{PP}$ consistently reflected progressive central hypovolemia during 


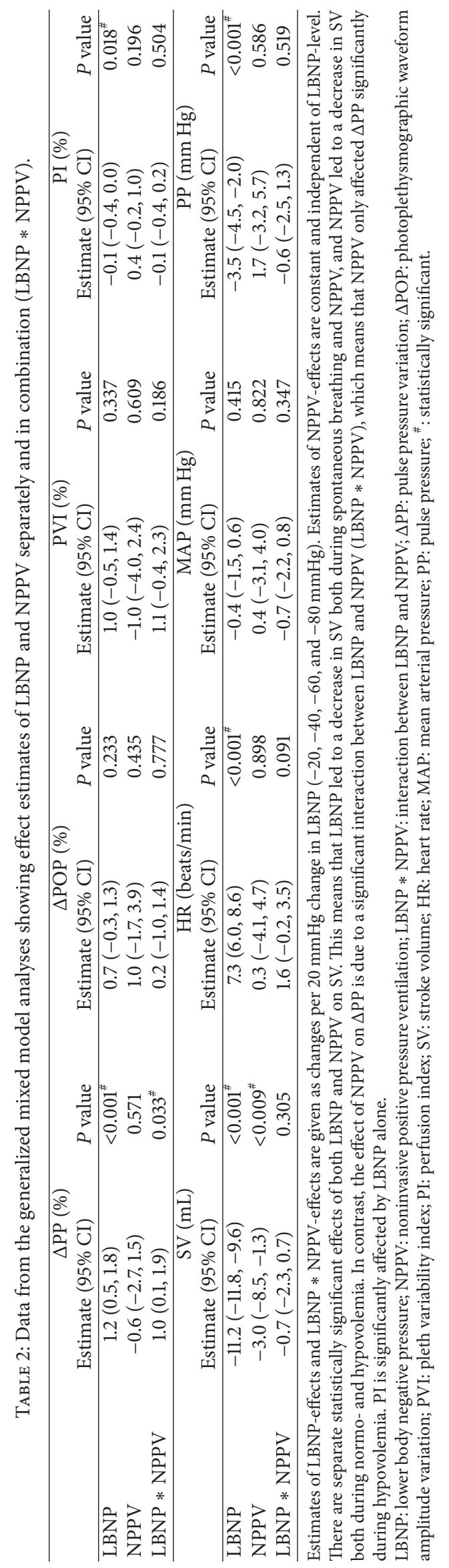




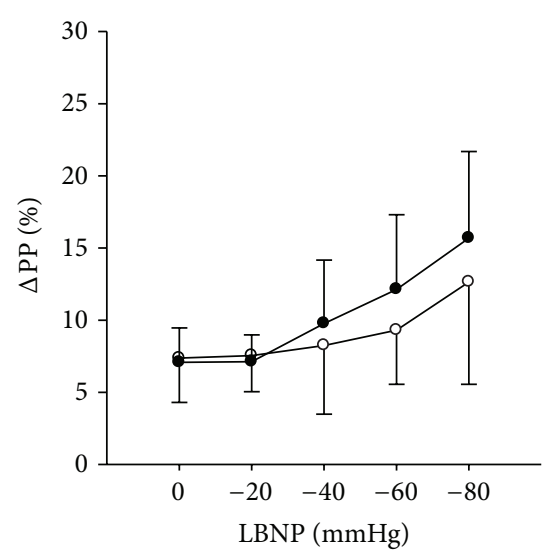

(a)

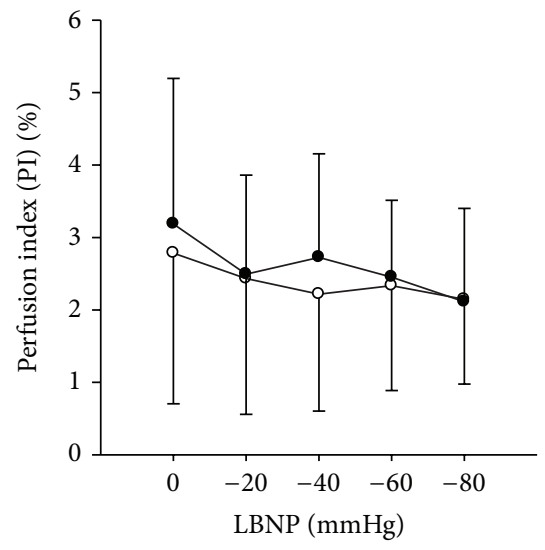

(d)

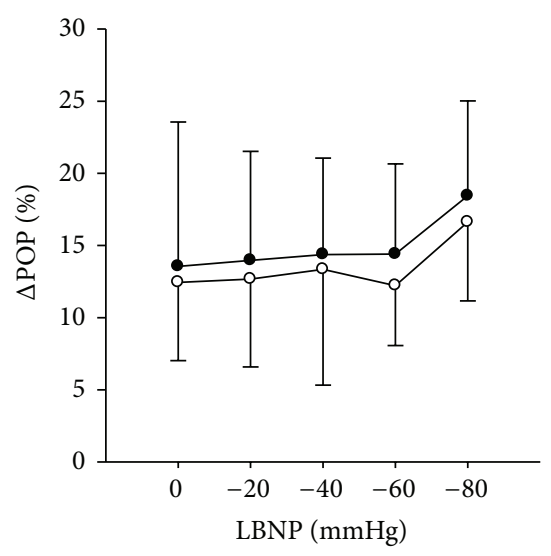

(b)

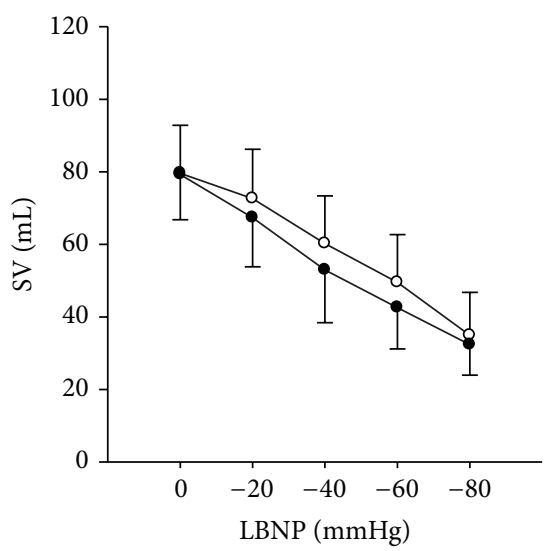

(e)

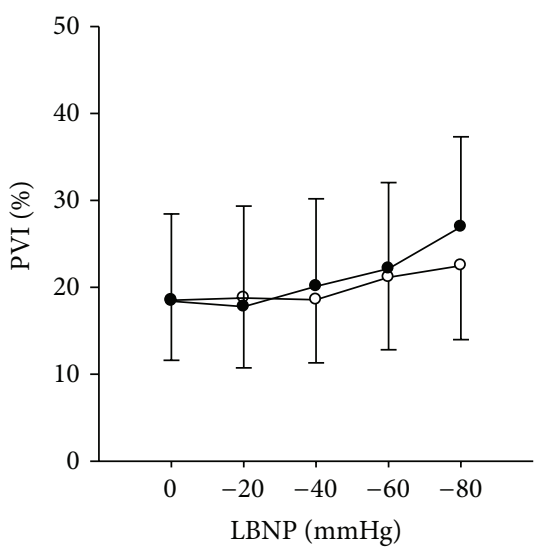

(c)

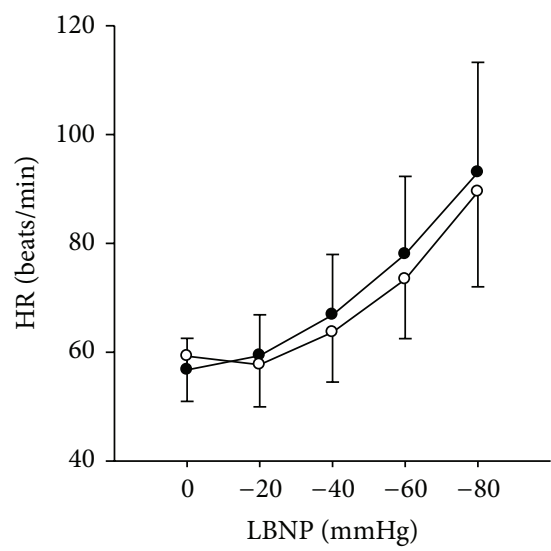

(f)

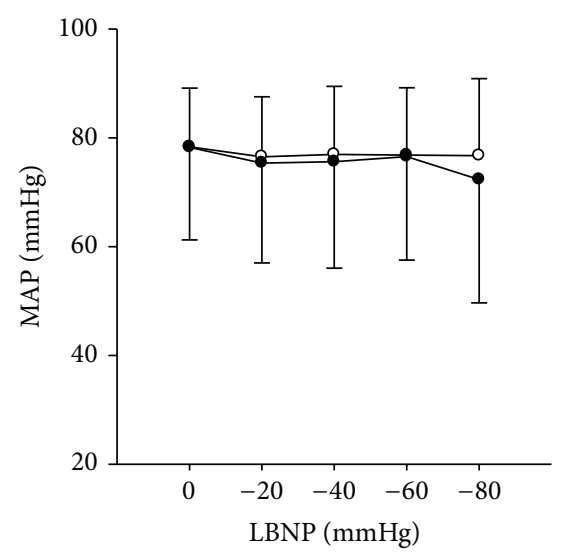

(g)

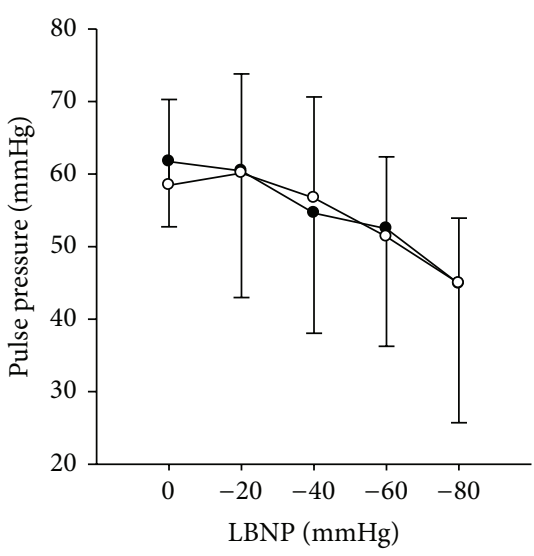

(h)

FIGURE 2: Line charts of mean values at each LBNP-level for $\triangle \mathrm{PP}, \triangle \mathrm{POP}, \mathrm{PVI}, \mathrm{PI}, \mathrm{SV}, \mathrm{HR}, \mathrm{MAP}$, and PP. Open circle: measurements during spontaneous breathing. Full circle: measurements during NPPV. 1 SD illustrated with one-sided error bars for clarity. $\triangle$ PP: respiratory variations in pulse pressure, $\triangle \mathrm{POP}$ : respiratory variations in the photoplethysmographic waveform amplitude, PVI: pleth variability index, PI: perfusion index, SV: stroke volume, HR: heart rate, MAP: mean arterial pressure, PP: pulse pressure, NPPV: noninvasive positive pressure ventilation, LBNP: lower body negative pressure.

NPPV. The application of NPPV accentuated the reduction in SV induced by LBNP. This accentuation was reflected by $\triangle \mathrm{PP}$ but not by $\triangle \mathrm{POP}$ or PVI. There were no associations between LBNP-levels and $\triangle \mathrm{POP}$ or PVI. Neither $\triangle \mathrm{POP}$ nor PVI changed significantly with the transition from spontaneous ventilation to NPPV.
4.1. SV and Pulse Pressure. The effects of LBNP on neurohumoral and sympathetic neural activity have been thoroughly described [10]. Increased vasomotor tone during early hypovolemia preserves systemic blood pressure and may in combination with increased heart rate mask reduced SV. We observed this compensatory response in the present 


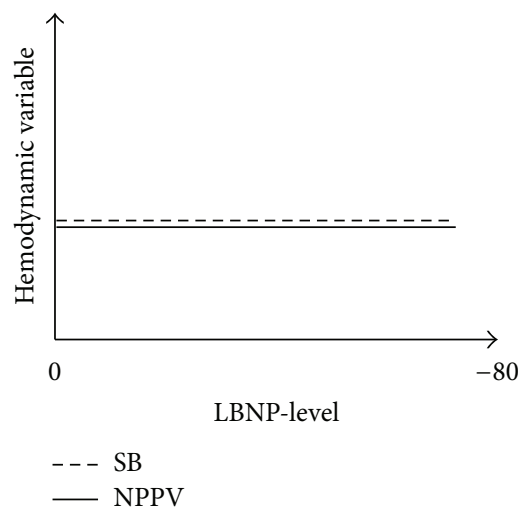

(a)

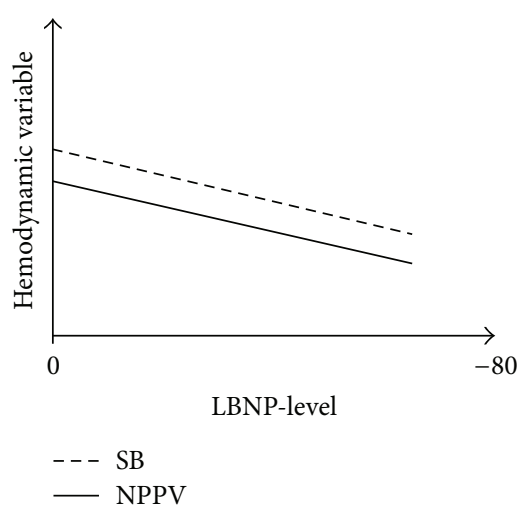

(b)

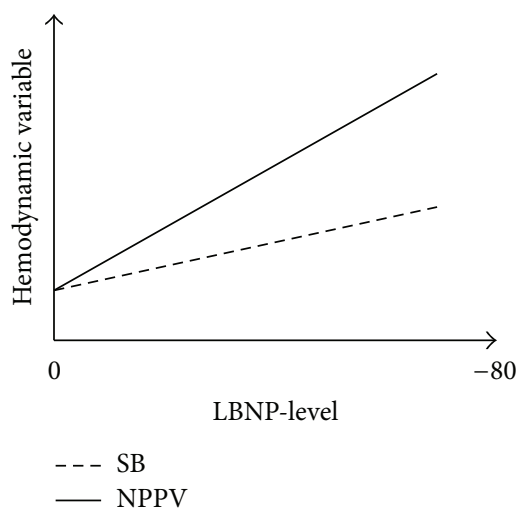

(c)

FIGURE 3: Illustration of different effects of LBNP and NPPV on hemodynamic variables. (a) No effect of LBNP or NPPV alone or in combination (for instance MAP), (b) independent effects of both LBNP and NPPV (stroke volume), and (c) independent effect of LBNP which increases in combination with NPPV (interaction; $\triangle \mathrm{PP}$ ). No effect of NPPV alone. LBNP: lower body negative pressure, NPPV: noninvasive positive pressure ventilation, SB: spontaneous breathing, MAP: mean arterial pressure, $\triangle \mathrm{PP}$ : pulse pressure variation.

study. Pulse pressure decreased with progressive LBNP-levels, but the decrease in pulse pressure occurred later than the decrease in SV. Similar results were reported in another study on spontaneously breathing volunteers undergoing progressive LBNP [14]. NPPV leads to an accentuated reduction in SV at all levels of LBNP except baseline. This is in accordance with the known impact of increased intrathoracic pressure on venous return during central hypovolemia [15]. The opposite effect was demonstrated by Ryan et al. [16] who found that breathing through an inspiratory threshold device preserved SV by reducing intrathoracic pressure, which increased LBNP-tolerance in healthy volunteers.

4.2. $\triangle P P, \triangle P O P, P V I$, and PI. Hemodynamic effects of controlled mechanical ventilation [10] and the ability of $\triangle \mathrm{PP}$ to reflect hypovolemia during mechanical ventilation have previously been demonstrated [17]. There are also studies indicating that dynamic variables like stroke volume variation and vena collapsibility index may be useful during spontaneous respiration [6]. Heart-lung interactions differ substantially between spontaneous breathing and mechanical ventilation, and insufficient changes in intrathoracic pressure due to low, irregular tidal volumes and irregular respiratory rates impede the use of respiratory induced variables to evaluate preload or preload dependency. Whereas mechanical inspiration reduces right ventricular filling and increases right ventricular afterload, spontaneous inspiration increases both right ventricular filling and right ventricular afterload. Deep inspiration could possibly induce sufficient changes in intrathoracic pressure to be reflected in SV and pulse pressure. Préau et al. [18] found that $\Delta \mathrm{PP}$ predicted fluid responsiveness in spontaneously breathing patients undergoing a deep inspiratory maneuver but with lower sensitivity than reported in studies on mechanically ventilated patients. Similarly, Soubrier et al. [19] tested volume responsiveness in spontaneously breathing patients and found that $\Delta \mathrm{PP}$ and variations in systolic blood pressure at baseline were significantly higher in responders than in nonresponders, but sensitivity was low. In a study on spontaneously breathing pigs $\triangle \mathrm{PP}$ was significantly higher during hypovolemia, but $\triangle \mathrm{PP}$ only predicted fluid responsiveness when an expiratory resistor was added [20]. Whereas none of these studies show that $\triangle \mathrm{PP}$ predicts fluid responsiveness with sufficient sensitivity, they indicate that pulse pressure variations may reflect hypovolemia during spontaneous breathing. This is in line with our findings, but in addition we show how $\triangle \mathrm{PP}$ increases following the transition from spontaneous breathing to NPPV. The substantial increase in $\triangle \mathrm{PP}$ during NPPV indicates that NPPV alters intrathoracic pressure similarly to invasive mechanical ventilation, provided compliance with the ventilator and minimal spontaneous breathing. Heenen et al. investigated dynamic variables in patients with spontaneous breathing movements and found that $\triangle \mathrm{PP}$ varied substantially at baseline with no significant differences between fluid responders and nonresponders, and areas under the receiver operating characteristics curves were low [7]. No standardization of respiratory rate, tidal volumes, or airway pressure was attempted in this study, which may in part explain their results. By manually filtering the data after recordings we were able to investigate the effects of NPPV alone, undisturbed by excessive spontaneous breathing.

There are conflicting reports on $\triangle \mathrm{POP}$ as an alternative to $\triangle \mathrm{PP}$ to reflect hypovolemia and predict fluid responsiveness $[8,21]$. Differences in vasomotor tone, measurement sites, and measurement methodology contribute to discrepant results. Promising studies on variables derived from the photoplethysmographic waveform have mainly been performed in stable patients during anesthesia, over short periods of time, and in the absence of sympathetic triggers such as advanced hypovolemia, surgery, pain, and stress $[8,22]$. In mechanically ventilated patients undergoing stepwise blood withdrawal, Pizov et al. [17] found that arterial waveform variables detected mild hypovolemia earlier and more consistently than photoplethysmographic variables. Interestingly, correlations between $\triangle \mathrm{POP}$ and $\triangle \mathrm{PP}$ improved with increasing hypovolemia of up to $20 \%$ of estimated blood volume, 
with $\triangle \mathrm{POP}$ showing the largest variability. These were all stable patients with no signs of circulatory failure. As shown in Table 1 and Figure 2, we found that $\triangle \mathrm{POP}$ increased with progressive LBNP, but due to large confidence intervals this increase was not statistically significant. Other studies have demonstrated wide limits of agreement between $\triangle \mathrm{PP}$ and $\triangle \mathrm{POP}$ during surgery and intensive care $[9,21,23,24]$. The physiology behind the photoplethysmographic signal is very complex, influenced by cardiac and autonomic as well as respiratory factors [25]. Due to rich innervation and large vascular plexuses the finger is very sensitive to vasomotion [26]. Using spectral analysis on both spontaneously breathing and mechanically ventilated patients, Shelley et al. [27] found a correlation between estimated blood loss and ventilatory effects in the ear signal but not in the finger signal. We believe that increased sympathetic activation leading to vasoconstriction is the main reason why $\triangle \mathrm{POP}$ to a lesser extent than $\triangle \mathrm{PP}$ reflected hypovolemia in our study and largely explains the discrepancy between this study and others performed on stable patients during normovolemia.

In addition to different measurement sites, the use of different features of the photoplethysmographic signal may explain conflicting reports. In a LBNP-model, McGrath et al. [28] studied the correlation between changes in SV and changes in pulse width, amplitude, and area of an unfiltered signal and found that it differed for all measurement sites. The lowest correlation was between changes in SV and pulse amplitude in the finger, the feature most commonly used when calculating respiratory changes in the photoplethysmographic waveform. Commercial finger pulse oximeters are frequently used in clinical practice and should be investigated. However, probes from different manufacturers could also contribute to different results as sensitivity and signal processing differ [29]. We obtained both pulse pressure and photoplethysmographic waveforms from noninvasive finger probes. The two measurement methods are based on different physiological principles (volume measurements in relatively large finger arteries versus absorption of infrared light from the tissues, primarily reflecting microcirculatory changes). It has previously been described how the complexity of the photoplethysmographic signal renders it more susceptible to "noise" which may be physiological or technological artifacts influencing the signal [29]. Our findings are in line with this explanation.

There was no significant relationship between PVI and LBNP-level in our study. Like other features of the photoplethysmographic waveform PVI depends on stable perfusion and vasomotion, as demonstrated in a recent study where changes in vasomotor tone were induced by norepinephrine [30]. Another study showed that PVI failed to predict fluid responsiveness in mechanically ventilated patients when PI was low $(<4)$, whereas pulse pressure variations reliably did [31]. The PI value depends on the fraction of infrared light returning from the measurement site and represents the ratio of pulsatile (pulsating blood) to nonpulsatile (nonpulsating blood, bone, and soft tissue) signal. PI is therefore affected by changes in vasomotor tone as this affects the ratio between pulsatile and nonpulsatile blood [32]. Increased vasomotor tone and low SV may explain the reduction in PI in the present study, and PI has been suggested as an early marker of the physiologic responses that occur during hypovolemia [33]. We experienced temporary loss of PVI signals in several subjects both during normo- and hypovolemia, although the photoplethysmographic waveform remained well defined. PI was $<1$ in all cases. The loss of PVI-signal has been described earlier [3].

4.3. Methodological Considerations. First, even with measurements on several levels in each subject our sample size is limited. It is however comparable to other studies using the LBNP-model $[34,35]$ and we were able to demonstrate significant effects of both hypovolemia and NPPV on SV and $\triangle \mathrm{PP}$. Second, we wanted to investigate the physiology in a completely noninvasive experimental model in order to avoid disturbing factors such as pain, agitation, and medication. Whereas pulse pressure variations are normally investigated using an invasive line, the Finapres technology has been validated for arterial pressure waveform analysis $[21,36]$ and blood pressure measurements [37]. Third, while the LBNPmodel allows investigation of reversible central hypovolemia, it is not suitable for conventional fluid responsiveness testing using intravenous fluid boluses. However, in this model fluid responsiveness is "tested" by termination of LBNP, which inevitably leads to increased preload and the restoration of hemodynamic variables [10]. Finally, application of NPPV to healthy, nonsedated subjects is challenging. The major issues were to minimize spontaneous breathing and leakage. High tidal volumes, PEEP $=0$ and IPPV-mode proved necessary to ensure compliance with NPPV and keep tidal volumes stable. Despite a compensatory increase in frequency, a reduction in tidal volumes to $8 \mathrm{~mL} / \mathrm{kg}$ increased spontaneous breathing efforts and leakage. Previous studies indicate that airway pressures, ventilation mode, tidal volumes, and lung compliance affect the hemodynamic effects of mechanical ventilation and thus the performance of dynamic variables [38-40]. Spontaneous breathing efforts varied in and between subjects and might have affected central hemodynamics. However, as we filtered the respiratory data obtained during NPPV after recording we identified periods with high, stable tidal volumes that were sufficient to result in measurable preload changes.

4.4. Conclusion. The main new finding in this experimental study on central hypovolemia is that $\triangle \mathrm{PP}$, but not $\triangle \mathrm{POP}$ or PVI, is significantly associated with LBNP-level in healthy volunteers during NPPV. Clinically, this implies that $\triangle \mathrm{PP}$ may be used to evaluate volume status in patients treated with NPPV. Further, clinical studies are needed to clarify the potential for $\triangle \mathrm{PP}$ in this setting.

\section{Conflict of Interests}

The authors declare no conflict of interests.

\section{Acknowledgment}

This work received departmental funding only. 


\section{References}

[1] P. E. Marik, X. Monnet, and J.-L. Teboul, "Hemodynamic parameters to guide fluid therapy," Annals of Intensive Care, vol. 1, article 1, 2011.

[2] M. Shamir, L. A. Eidelman, Y. Floman, L. Kaplan, and R. Pizov, "Pulse oximetry plethysmographic waveform during changes in blood volume," British Journal of Anaesthesia, vol. 82, no. 2, pp. 178-181, 1999.

[3] M. Cannesson, B. Delannoy, A. Morand et al., "Does the pleth variability index indicate the respiratory-induced variation in the plethysmogram and arterial pressure waveforms?" Anesthesia and Analgesia, vol. 106, no. 4, pp. 1189-1194, 2008.

[4] J.-L. Teboul and X. Monnet, "Detecting volume responsiveness and unresponsiveness in intensive care unit patients: two different problems, only one solution," Critical Care, vol. 13, no. 4, p. 175, 2009.

[5] X. Monnet, D. Osman, C. Ridel, B. Lamia, C. Richard, and J.-L. Teboul, "Predicting volume responsiveness by using the endexpiratory occlusion in mechanically ventilated intensive care unit patients," Critical Care Medicine, vol. 37, no. 3, pp. 951-956, 2009.

[6] M. J. Lanspa, C. K. Grissom, E. L. Hirshberg, J. P. Jones, and S. M. Brown, "Applying dynamic parameters to predict hemodynamic response to volume expansion in spontaneously breathing patients with septic shock," Shock, vol. 39, no. 2, pp. 155-160, 2013.

[7] S. Heenen, D. de Backer, and J.-L. Vincent, "How can the response to volume expansion in patients with spontaneous respiratory movements be predicted?" Critical Care, vol. 10, no. 4, article R102, 2006.

[8] M. Cannesson, Y. Attof, P. Rosamel et al., "Respiratory variations in pulse oximetry plethysmographic waveform amplitude to predict fluid responsiveness in the operating room," Anesthesiology, vol. 106, no. 6, pp. 1105-1111, 2007.

[9] R. H. Thiele, D. A. Colquhoun, K. T. Forkin, and M. E. Durieux, "Assessment of the agreement between photoplethysmographic and arterial waveform respiratory variation in patients undergoing spine surgery," Journal of Medical Engineering \& Technology, vol. 37, no. 7, pp. 409-415, 2013.

[10] W. H. Cooke, K. L. Ryan, and V. A. Convertino, "Lower body negative pressure as a model to study progression to acute hemorrhagic shock in humans," Journal of Applied Physiology, vol. 96, no. 4, pp. 1249-1261, 2004.

[11] V. A. Convertino, W. H. Cooke, and J. B. Holcomb, "Arterial pulse pressure and its association with reduced stroke volume during progressive central hypovolemia," Journal of Trauma, vol. 61, no. 3, pp. 629-634, 2006.

[12] J. Hisdal, K. Toska, and L. Walløe, "Design of a chamber for lower body negative pressure with controlled onset rate," Aviation Space and Environmental Medicine, vol. 74, no. 8, pp. 874-878, 2003.

[13] F. Michard, "Changes in arterial pressure during mechanical ventilation," Anesthesiology, vol. 103, no. 2, pp. 419-428, 2005.

[14] A. T. Reisner, D. Xu, K. L. Ryan, V. A. Convertino, C. A. Rickards, and R. Mukkamala, "Monitoring non-invasive cardiac output and stroke volume during experimental human hypovolaemia and resuscitation," British Journal of Anaesthesia, vol. 106, no. 1, pp. 23-30, 2011.

[15] M. R. Pinsky, "Cardiovascular issues in respiratory care," Chest, vol. 128, no. 5, supplement 2, pp. 592S-597S, 2005.
[16] K. L. Ryan, W. H. Cooke, C. A. Rickards, K. G. Lurie, and V. A. Convertino, "Breathing through an inspiratory threshold device improves stroke volume during central hypovolemia in humans," Journal of Applied Physiology, vol. 104, no. 5, pp. 14021409, 2008.

[17] R. Pizov, A. Eden, D. Bystritski, E. Kalina, A. Tamir, and S. Gelman, "Arterial and plethysmographic waveform analysis in anesthetized patients with hypovolemia," Anesthesiology, vol. 113, no. 1, pp. 83-91, 2010.

[18] S. Préau, F. Dewavrin, V. Soland et al., "Hemodynamic changes during a deep inspiration maneuver predict fluid responsiveness in spontaneously breathing patients," Cardiology Research and Practice, vol. 2012, Article ID 191807, 8 pages, 2012.

[19] S. Soubrier, F. Saulnier, H. Hubert et al., "Can dynamic indicators help the prediction of fluid responsiveness in spontaneously breathing critically ill patients?" Intensive Care Medicine, vol. 33, no. 7, pp. 1117-1124, 2007.

[20] M. K. Dahl, S. T. Vistisen, J. Koefoed-Nielsen, and A. Larsson, "Using an expiratory resistor, arterial pulse pressure variations predict fluid responsiveness during spontaneous breathing: an experimental porcine study," Critical Care, vol. 13, no. 2, article R39, 2009.

[21] H. Solus-Biguenet, M. Fleyfel, B. Tavernier et al., "Noninvasive prediction of fluid responsiveness during major hepatic surgery," British Journal of Anaesthesia, vol. 97, no. 6, pp. 808816, 2006.

[22] M. Cannesson, O. Desebbe, P. Rosamel et al., "Pleth variability index to monitor the respiratory variations in the pulse oximeter plethysmographic waveform amplitude and predict fluid responsiveness in the operating theatre," British Journal of Anaesthesia, vol. 101, no. 2, pp. 200-206, 2008.

[23] L. Ø. Høiseth, I. E. Hoff, Ø. Skare, K. A. Kirkebøen, and S. A. Landsverk, "Photoplethysmographic and pulse pressure variations during abdominal surgery," Acta Anaesthesiologica Scandinavica, vol. 55, no. 10, pp. 1221-1230, 2011.

[24] S. A. Landsverk, L. O. Hoiseth, P. Kvandal, J. Hisdal, O. Skare, and K. A. Kirkeboen, "Poor agreement between respiratory variations in pulse oximetry photoplethysmographic waveform amplitude and pulse pressure in intensive care unit patients," Anesthesiology, vol. 109, no. 5, pp. 849-855, 2008.

[25] L. M. Nilsson, "Special article: respiration signals from photoplethysmography," Anesthesia \& Analgesia, vol. 117, no. 4, pp. 859-865, 2013.

[26] A. A. Awad, M. A. M. Ghobashy, W. Ouda, R. G. Stout, D. G. Silverman, and K. H. Shelley, "Different responses of ear and finger pulse oximeter wave form to cold pressor test," Anesthesia and Analgesia, vol. 92, no. 6, pp. 1483-1486, 2001.

[27] K. H. Shelley, D. H. Jablonka, A. A. Awad, R. G. Stout, H. Rezkanna, and D. G. Silverman, "What is the best site for measuring the effect of ventilation on the pulse oximeter waveform?" Anesthesia and Analgesia, vol. 103, no. 2, pp. 372377, 2006.

[28] S. P. McGrath, K. L. Ryan, S. M. Wendelken, C. A. Rickards, and V. A. Convertino, "Pulse oximeter plethysmographic waveform changes in awake, spontaneously breathing, hypovolemic volunteers," Anesthesia and Analgesia, vol. 112, no. 2, pp. 368-374, 2011.

[29] X. Monnet, B. Lamia, and J.-L. Teboul, "Pulse oximeter as a sensor of fluid responsiveness: do we have our finger on the best solution?” Critical Care, vol. 9, no. 5, pp. 429-430, 2005.

[30] X. Monnet, L. Guerin, M. Jozwiak et al., "Pleth variability index is a weak predictor of fluid responsiveness in patients receiving 
norepinephrine," British Journal of Anaesthesia, vol. 110, no. 2, pp. 207-213, 2013.

[31] O. Broch, B. Bein, M. Gruenewald et al., "Accuracy of the pleth variability index to predict fluid responsiveness depends on the perfusion index," Acta Anaesthesiologica Scandinavica, vol. 55, no. 6, pp. 686-693, 2011.

[32] A. P. Lima, P. Beelen, and J. Bakker, "Use of a peripheral perfusion index derived from the pulse oximetry signal as a noninvasive indicator of perfusion," Critical Care Medicine, vol. 30, no. 6, pp. 1210-1213, 2002.

[33] M. E. van Genderen, S. A. Bartels, A. Lima et al., "Peripheral perfusion index as an early predictor for central hypovolemia in awake healthy volunteers," Anesthesia \& Analgesia, vol. 116, no. 2, pp. 351-356, 2013.

[34] J. C. Puyana, M. Pinsky, U. Leuenberger, and J. Berkow, "Modelbased prediction of autoregulatory exhaustion in response to lower-body negative pressure-induced shock," Journal of Trauma and Acute Care Surgery, vol. 75, no. 2, supplement 2, pp. S190-S196, 2013.

[35] L. M. Nilsson, D. M. Lindenberger, and R. G. Hahn, "The effect of positive end-expiratory pressure and tripled tidal volume on pleth variability index during hypovolaemia in conscious subjects: a volunteer study," European Journal of Anaesthesiology, vol. 30, no. 11, pp. 671-677, 2013.

[36] B. Lansdorp, D. Ouweneel, A. De Keijzer, J. G. Van Der Hoeven, J. Lemson, and P. Pickkers, "Non-invasive measurement of pulse pressure variation and systolic pressure variation using a finger cuff corresponds with intra-arterial measurement," British Journal of Anaesthesia, vol. 107, no. 4, pp. 540-545, 2011.

[37] J. R. Martina, B. E. Westerhof, J. van Goudoever et al., "Noninvasive continuous arterial blood pressure monitoring with nexfin," Anesthesiology, vol. 116, no. 5, pp. 1092-1103, 2012.

[38] F. Vallée, J. C. M. Richard, A. Mari et al., "Pulse pressure variations adjusted by alveolar driving pressure to assess fluid responsiveness," Intensive Care Medicine, vol. 35, no. 6, pp. 1004-1010, 2009.

[39] D. de Backer, S. Heenen, M. Piagnerelli, M. Koch, and J.-L. Vincent, "Pulse pressure variations to predict fluid responsiveness: influence of tidal volume," Intensive Care Medicine, vol. 31, no. 4, pp. 517-523, 2005.

[40] X. Monnet, A. Bleibtreu, A. Ferré et al., "Passive leg-raising and end-expiratory occlusion tests perform better than pulse pressure variation in Patients with low respiratory system compliance," Critical Care Medicine, vol. 40, no. 1, pp. 152-157, 2012. 


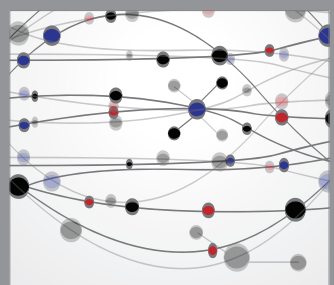

The Scientific World Journal
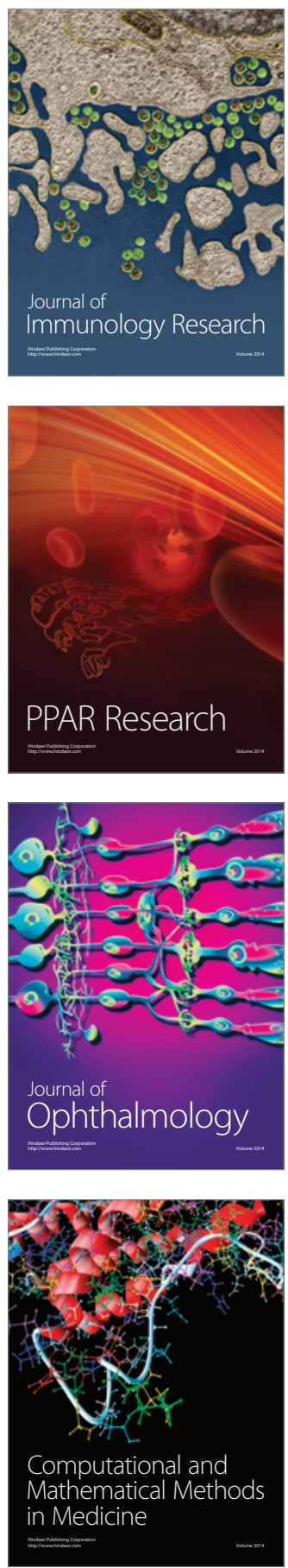

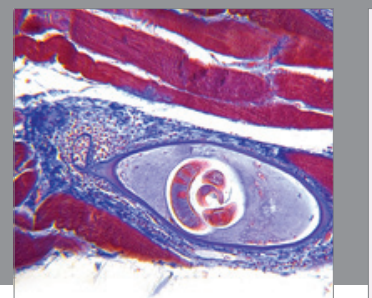

Gastroenterology

Research and Practice
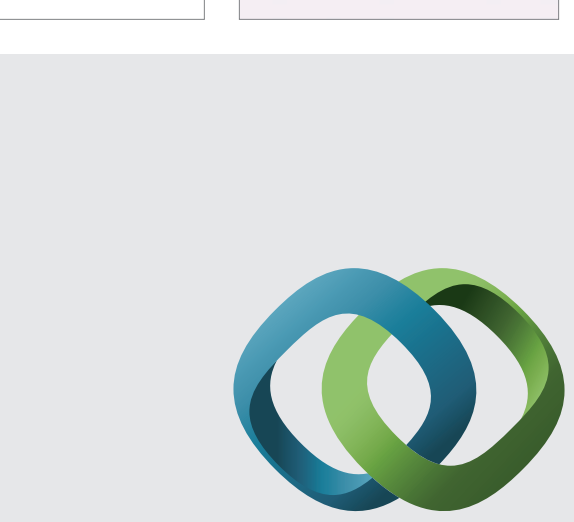

\section{Hindawi}

Submit your manuscripts at

http://www.hindawi.com
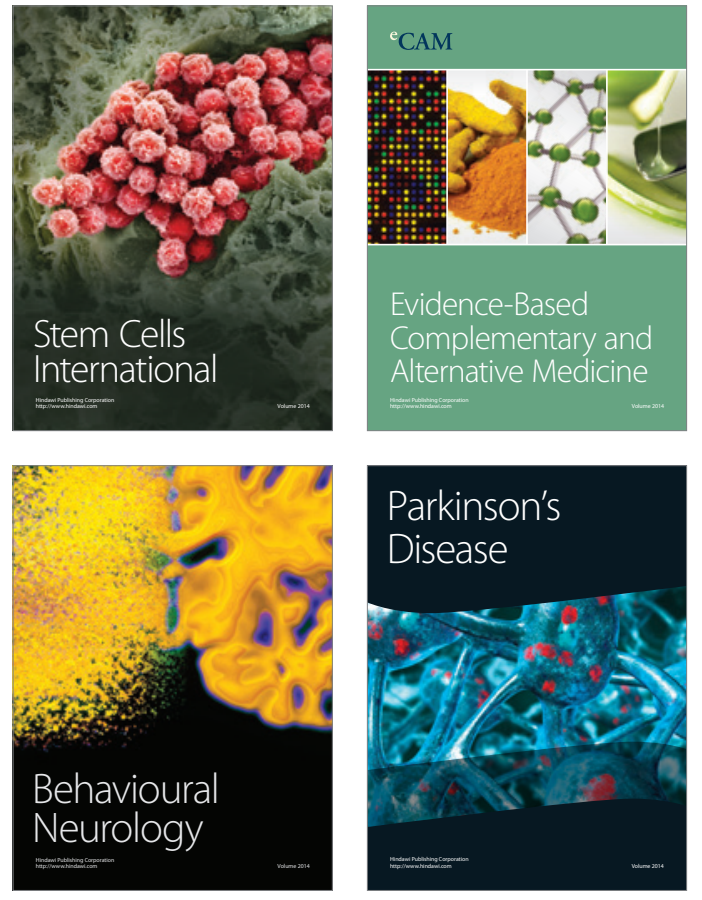
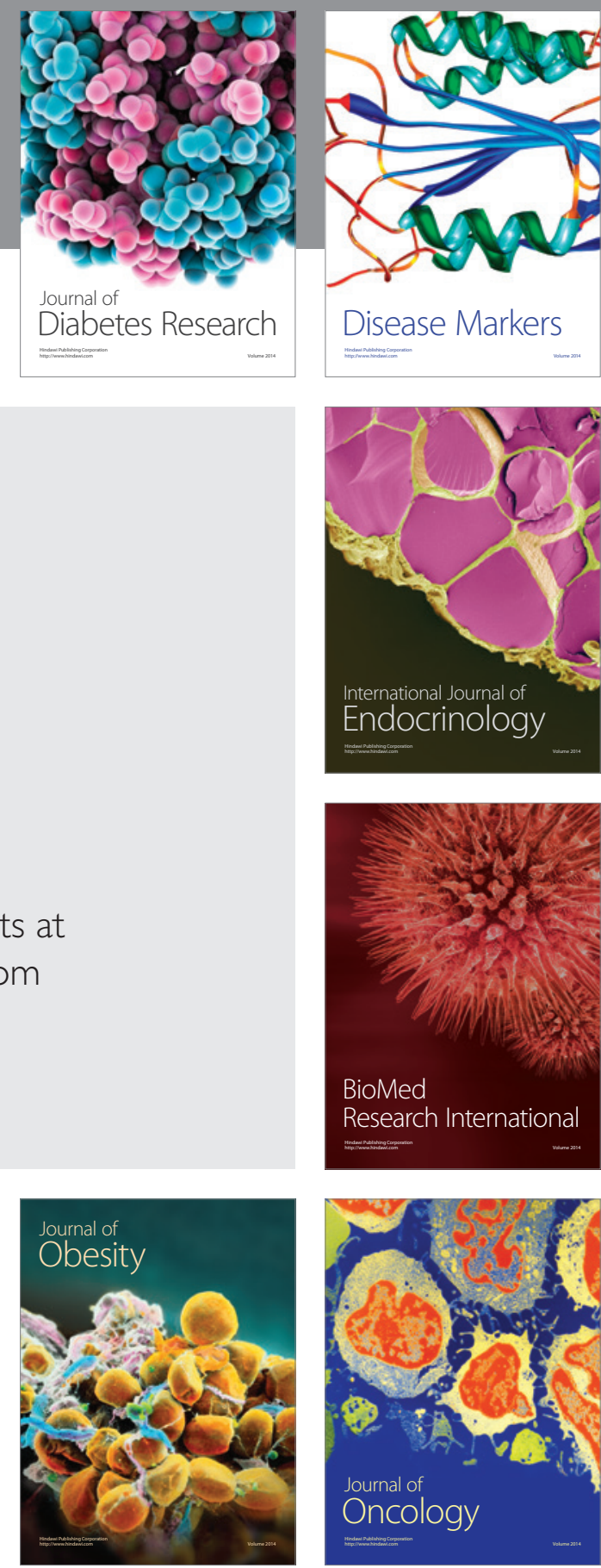

Disease Markers
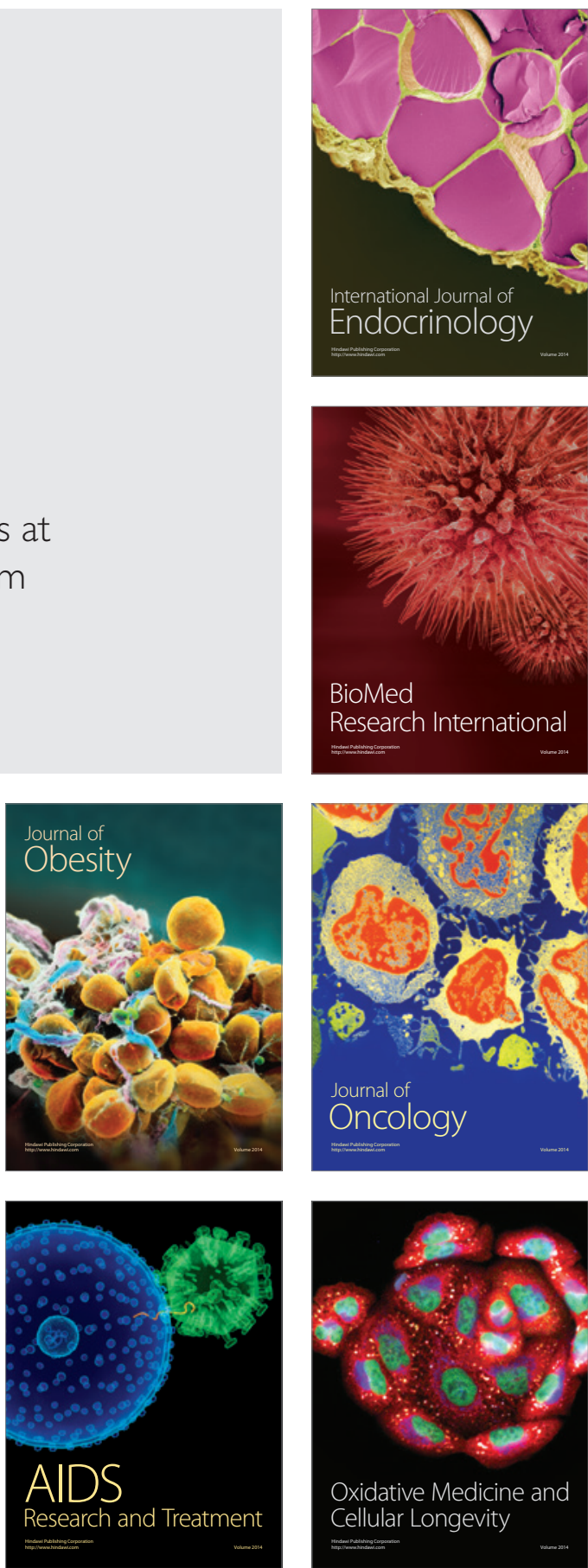